\title{
Myocardial Boundary Extraction Using Coupled Active Contours
}

\author{
Luuk Spreeuwers $^{1}$, Marcel Breeuwer ${ }^{2}$ \\ ${ }^{1}$ Image Sciences Institute, University Medical Center Utrecht, Netherlands \\ ${ }^{2}$ Medical IT - Advanced Development, Philips Medical Systems Best, Netherlands
}

\begin{abstract}
Automatic extraction of object boundaries in medical images using active contours is a popular research item. A problem with active contours is that they may fail to give correct results in cases of low contrast, noise or the neighbourhood of other edges. If there exists a geometrical relation between several boundaries this may be exploited to improve the robustness of active contours. In this way a set of coupled active contours results. This paper describes the simultaneous extraction of epiand endocardial boundaries using a set of coupled active contours in MR short axis cine recordings.
\end{abstract}

\section{Introduction}

Cine MRI acquisitions, in particular multi-slice shortaxis acquisitions of the left ventricle, have proven their usefulness for the determination of ventricular volume, myocardial mass and other global and local function parameters, like e.g. local wall thickness. These 4$\mathrm{D}$ datasets contain a number of short-axis slices through the heart for multiple phases of the cardiac cycle. In order to determine the cardiac parameters, the epi- and endocardial boundaries must be traced. Using Simpson's rule stroke volume, ejection fraction etc. can be derived from the contours. Manual tracing of the contours in all images is a rather labour intensive task, therefore, methods were proposed to automatically or semi-automatically extract the contours from short axis images, see e.g. $[1,2]$. Problems that complicate automatic contour

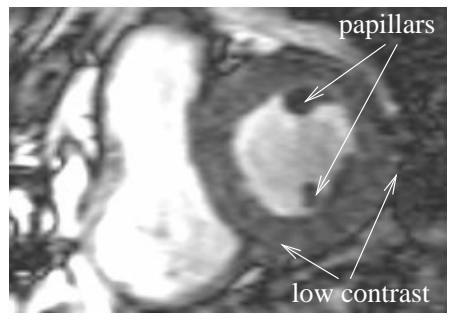

Figure 1. Papillary muscles and low contrast complicate cardiac segmentation. extraction are low contrast between the myocardium and other tissues and the papillary muscles, that for wallthickness measurements must be placed inside and for left ventricular volume measurements outside the endocardial boundary. Generally, the epi- and endocardial boundaries are treated independently and the two contours are extracted separately. In this paper a coupled active contour approach is proposed that exploits the fact that the epi- and endocardial boundaries are not independent and extracts both contours simultaneously.

\section{Active contours}

Active contour models are used to fit a smooth or otherwise shape-constraint contour to local image information, e.g. the gradient. There exist numerous implementations of this basic idea like snakes [3], active shape models [4], discrete dynamic contour models [5] and level sets [6]. Generally, the local image information used, is in the direction perpendicular to the contour, e.g. the derivative of a 1-D intensity profile across the contour. The contour is then positioned such that a trade-off consists between the shape-constraints (e.g. a smoothness constraint) and the distance to the maximum of the derivative (see fig.2). Several publications have

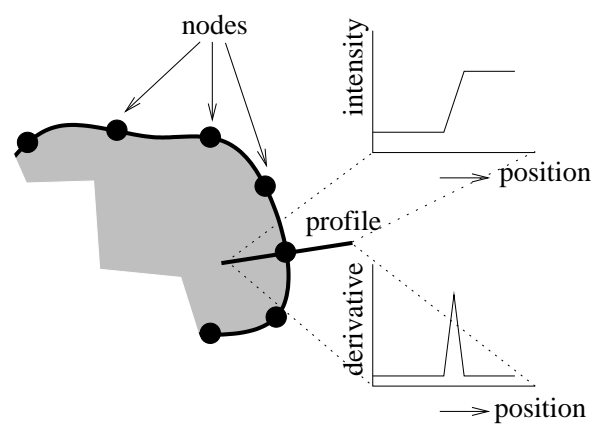

Figure 2. Basic idea of an active contour: a smooth contour is placed as close as possible to the maximum gradient of the 1-D profiles of the nodes.

appeared about applying active contour techniques for extraction of epi- and endocardial boundaries in short axis recordings of the heart, see e.g. [1,2]. These methods 
usually treat the epi- and endocardial contours separately, although the active shape model described in [1] implicitly uses the geometric relation between the contours, because of the statistical shape constraints.

\section{Coupled active contours}

The difference between coupled active contours and a simple active contour is that for coupled active contours there are two nodes (or more) on an intensity profile, whereas there is only one for the simple active contour approach. Thus the positions of both contours are estimated simultaneously. However, fitting the intensity profile is also more complicated. Generally, for a simple active contour approach, translation suffices for the fitting process. For a coupled active contour approach, the distance between the two nodes on the intensity profile may have to be adjusted, which implies that some kind of scaling must be used as well. Because more local characteristics are captured in the intensity profiles (two edges and their relative positions) it is to be expected that the coupled active contour method is more robust. Also the inner contour will never be attracted to edges outside the outer boundary and vice versa, i.e. the topology is always preserved. Fig. 3 shows a set of coupled contours, the aligned nodes on the contours and the profiles. In this case the profiles are not perpendicular to the contours, but radiating from the centre of gravity of the inner contour.

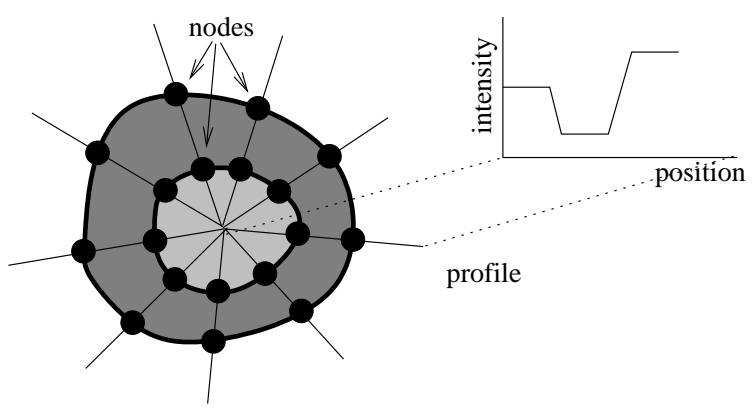

Figure 3. Coupled contour with two nodes per intensity profile. By estimating the positions of the node-pairs, the inner and outer contours are extracted simultaneously.

In order to be able to compare the intensity profiles, they must have the same number of samples. Scaling and translation of the profiles was realised by resampling the profiles with a fixed number of samples between each pair of nodes (see fig. 4). The number of samples may differ between different pairs of nodes. To find the correct position of the nodes on a profile, for each combination of the positions of the nodes, the profile is resampled and compared to a reference profile (e.g. a double edge model). The correct position of the nodes results in the smallest difference between the two profiles. Smoothing was realised by an iterative process where significantly deviating node displacements were substituted by the average displacements of neighbouring nodes on the same contour.

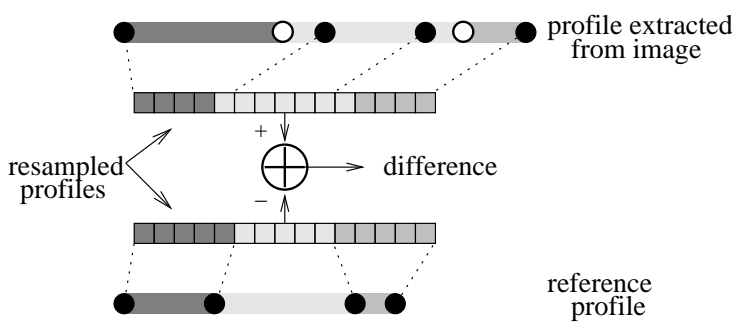

Figure 4. Resampling of intensity profiles. Both profiles are resampled with a fixed number of samples between the nodes. If the nodes on the profile at the top are positioned correctly (open circles), the difference between the resampled profiles vanishes.

\section{Propagating through a time series}

As mentioned before, the presence of the papillary muscles complicates the segmentation. Generally, one would like the papillary muscles to lie inside the endocardial boundary. However, often, there is virtually no contrast between the papillary muscles and the myocardium, which makes straightforward gradient-based segmentation impossible. Therefore, we chose to start from a good (manual) initial segmentation and initialise the intensity profiles from this segmentation. The epiand endocardial contours are then propagated through the whole time series by scaling and translating these initial profiles such that they best fit the actual image data in the subsequent image. In this way for each node on the profiles the correct position is found in the new image. After each propagation step, the just propagated contours and corresponding profiles become the reference contours and reference profiles for the next propagation step. This approach has the advantage that large changes in image characteristics can be handled. However, errors are propagated as well and may increase during propagation.

\section{Experiments and results}

A number of experiments were performed to validate and evaluate the coupled contour propagation method. These included experiments with synthetic images and patient data. Qualitative experiments were performed with many different datasets. The coupled active contour approach appeared very robust and according to visual inspection the results were good. Quantitative comparisons between 
propagated and manual segmentations were carried out for contour position and dynamic wall-thickness and volume measurements.

\subsection{Validation using synthetic images}

Fig. 5 shows the results of a coupled and a simple active contour for an image sequence with two interfering edges. The simple active contour (black) is disturbed by the
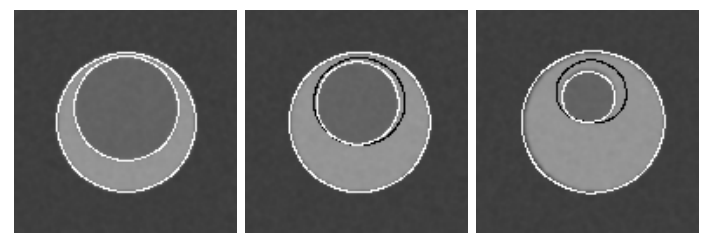

Figure 5. Results of the coupled active contours (white) and a simple active contour (black) for a synthetic image sequence.

presence of a second edge and the inaccuracy of the edge estimation is enhanced by the propagation. The coupled active contour (white) correctly handles the interfering edges.

\subsection{Evaluation using patient data}

A software tool was developed for the Philips EasyVision environment that allows both manual tracing of contours and automatic propagation from an initial set of contours. The datasets we used for evaluation were recorded using balanced FFE on a Philips Gyroscan MR scanner at the German Heart Institute in Berlin, Germany and at the University Medical Centre in Utrecht, Netherlands. Datasets I-III consisted of 3 slices and datasets IV and $\mathrm{V}$ of 10 resp. 12 slices with a slice thickness of $8 \mathrm{~mm}$. The in-plane image resolution was $256 \times 256$ pixels and the field of view ranged from $350 \times 350$ to $400 \times 400 \mathrm{~mm}$. All datasets contained 20 phases. Manual tracing of the epiand endocardial contours takes about 30 seconds per frame or about half an hour for a dataset with 3 slices. Manual tracing of complete datasets turned out to be a very tiring task. Propagation of the contours of a single slice through 20 phases takes about 2 seconds on a modern PC. A 3-slice dataset could easily be segmented within 3 minutes using propagation including the manual segmentation of the first phase. The results of the propagation for one of the datasets are shown in fig. 6.

Note that the presence of the papillary muscles causes no problems to the coupled active contours approach, because it does not use the gradient in the images, but rather the reference intensity profiles initialised using the manual segmentation to position the contours.

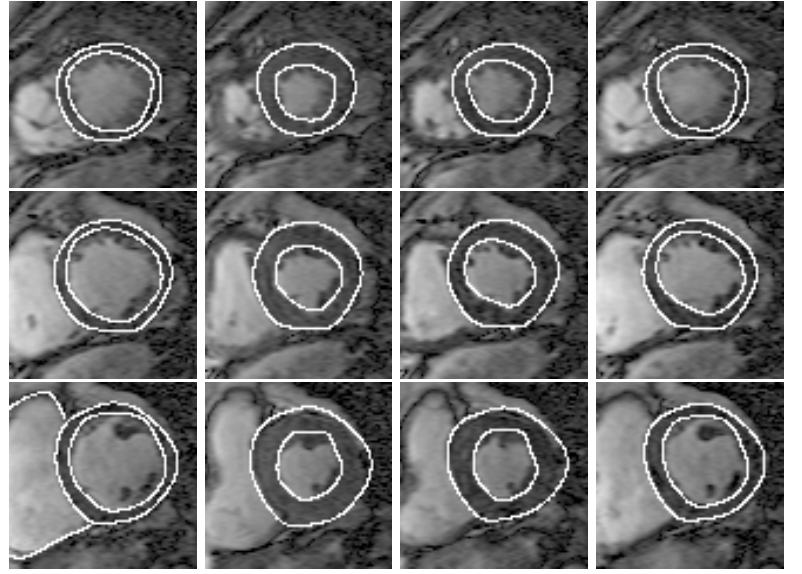

Figure 6. Coupled active contour segmentation results for a dataset of 3 slices and 20 phases (only phases 1/8/12/20 are shown). The first phase is segmented manually.

\subsection{Contour position accuracy}

For quantitative evaluation, datasets I-III were segmented twice by a single observer. Dataset IV was also segmented by a second observer. The results were compared to those obtained using propagation. From the results in table 1

\begin{tabular}{|l|l|l|r|r|r|r|r|}
\cline { 3 - 8 } \multicolumn{2}{c|}{} & \multicolumn{2}{c|}{ endo contour } & \multicolumn{2}{l|}{ epi contour } & \multicolumn{2}{l|}{} \\
\cline { 3 - 8 } \multicolumn{2}{c|}{} & av. & max. & av. & max. & $\mathrm{EF}_{p}$ & $\mathrm{EF}_{m}$ \\
\hline I & obs1 & 1.2 & 6.5 & 0.8 & 5.2 & 56 & 63 \\
& obs1 & 1.2 & 4.7 & 0.8 & 4.6 & 62 & 62 \\
& intra & 0.6 & 2.9 & 0.6 & 2.9 & & \\
\hline II & obs1 & 1.0 & 4.5 & 0.8 & 4.6 & 38 & 31 \\
& obs1 & 1.1 & 6.1 & 0.9 & 3.7 & 38 & 52 \\
& intra & 0.6 & 3.2 & 0.5 & 3.4 & & \\
\hline III & obs1 & 1.1 & 4.2 & 0.8 & 3.7 & 65 & 53 \\
& obs1 & 1.0 & 4.2 & 0.7 & 4.6 & 66 & 57 \\
& intra & 0.6 & 2.6 & 0.6 & 4.5 & & \\
\hline IV & obs1 & 0.6 & 4.6 & 0.5 & 4.0 & 51 & 44 \\
& obs1 & 0.4 & 6.3 & 0.3 & 3.6 & 48 & 36 \\
& obs2 & 1.1 & 5.4 & 0.8 & 4.1 & 52 & 43 \\
& intra & 0.2 & 3.7 & 0.2 & 2.8 & & \\
& inter & 0.4 & 4.9 & 0.4 & 3.5 & & \\
\hline V & obs1 & 0.9 & 4.1 & 0.7 & 4.6 & 54 & 51 \\
\hline
\end{tabular}

Table 1. Average and maximum differences between propagated and manual segmentations and ejection fractions for 5 datasets. All differences are given in pixels.

the conclusion can be drawn that the average difference between manual and propagated contours is in the order of 1 pixel and almost twice as large as the intra-observer differences, but quite comparable with the inter-observer differences. The maximum differences for the endocardial 
contour occurred near end systole, when the size of the left ventricle is minimal. This is in accordance with results from Sechtem [7] and Semelka [8] who found that the endsystolic left ventricular volume was the most difficult to measure parameter and reported inter-observer variabilities of $10 \%$ and larger. The ejection fractions obtained using the propagation method $\left(\mathrm{EF}_{p}\right)$ was generally a bit higher than those obtained using manual segmentations $\left(\mathrm{EF}_{m}\right)$.

\subsection{Dynamic wall-thickness and volume measurements}

Especially interesting are dynamic wall-thickness and volume measurements, that first become possible if a complete segmentation of all phases is available. It appeared that the manual segmentations show many sudden jumps of the contours between subsequent phases, which makes studying dynamic wall-thickness behaviour very difficult. The contours produced by the coupled active contours approach show a much smoother behaviour and clearly show the contraction of the left ventricle and the myocardium. Fig.7 shows a comparison of dynamic wall-thickness (left) and left ventricle volume (right) measurements for manual and propagated segmentations. The wall-thickness graph represents the wall-thickness at
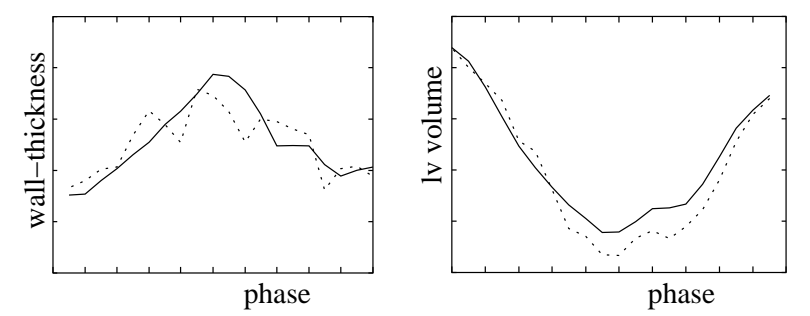

Figure 7. Comparison of dynamic Wall-thickness (left) and left ventricular volume (right) behaviour for manual (dotted lines) and propagated (solid lines) segmentations.

a single position in the myocardium. The left ventricular volume graph represents the sum of the volumes of the 3 slices. Because of this summation and the integration to calculate the volume, the irregular behaviour of the volume measurement is less than that of the wall-thickness.

\section{Conclusions and further work}

A new method for segmentation of endo- and epicardial boundaries in cine short-axis MR recordings is presented that exploits the relation between the endo- and epicardial boundaries. This coupled active contours approach extracts both contours simultaneously. First experiments show the coupled active contour approach to be robust and the first results are encouraging. The basic ideas of a coupled active contour should be applicable to other active contour/surface/object approaches like active shape models as well.

Currently, work is focusing on the propagation through slices, which is more complicated, because the differences between slices are much larger than between the phases of a single slice, and more extensive evaluation. Also, we consider extension of the work to a 3-D coupled active surface approach.

\section{Acknowledgements}

Image material used in this study was kindly supplied to us by the German Heart Institute, Augustenburger Platz 1, Berlin, Germany and the University Medical Centre, Utrecht, the Netherlands.

\section{References}

[1] van der Geest RJ, Reiber JHC. Quantifi cation in cardiac MRI. Journal of Magnetic Resonance Imaging 1999;10:602-608.

[2] Rangenath S. Contour extraction from cardiac MRI studies using snakes. IEEE Transactions on Medical Imaging 1995; 14(2):309-318.

[3] Kass M, Witkin A, Terzopoulos D. Snakes: Active contour models. International Journal of Computer Vision 1988;321331.

[4] Cootes TF, Hill A, Taylor CJ, Haslam J. The use of active shape models for locating structures in medical images. Image and Vision Computing 1994;12(6):355-366.

[5] Lobregt A, Viergever MA. A discrete dynamic contour model. IEEE Transactions on Medical Imaging 1995;14(1):12-24.

[6] Sethian JA. Level Set Methods and Fast Marching Methods: Evolving Interfaces in Computational Geometry, Fluid Mechanics, Computer Vision and Materials Science. Cambridge University Press, 1999.

[7] Sechtem U, Pflugfelder PW, Gould RG, Cassidy MM, Higgins CB. Measurement of right and left ventricular volumes in healthy individuals with cine MR imaging. Radiology 1987; 163:697-702.

[8] Semelka RC, Tomei E, Wagner S. Normal left ventricular dimensions and function: interstudy reproducibility of measurements with cine MR imaging. Radiology 1990; 174:763-768.

Address for correspondence:

Luuk Spreeuwers

Image Sciences Institute / University Medical Center Utrecht Heidelberglaan $100 / 3584$ CX Utrecht / Netherlands tel./fax: ++31-30-250-6682/251-3399

luuk@isi.uu.nl 